\title{
Regional Differences in Antithrombotic Treatment for Atrial Fibrillation: Insights from the GLORIA-AF Phase II Registry
}

Michał Mazurek ${ }^{1,2}$ Menno V. Huisman ${ }^{3} \quad$ Kenneth J. Rothman ${ }^{4}$ Miney Paquette ${ }^{5}$ Christine Teutsch 6 Hans-Christoph Diener ${ }^{7}$ Sergio J. Dubner ${ }^{8}$ Jonathan L. Halperin ${ }^{9}$ Chang Sheng Ma ${ }^{10}$ Kristina Zint $^{11}$ Amelie Elsaesser ${ }^{12}$ Shihai Lu ${ }^{13}$ Gregory Y. H. Lip ${ }^{1,14}$ on behalf of the GLORIA-AF Investigators

${ }^{1}$ Institute of Cardiovascular Sciences, University of Birmingham, Birmingham, United Kingdom

2 Department of Cardiology, Congenital Heart Diseases and Electrotherapy, Silesian Medical University, Silesian Centre for Heart Diseases, Zabrze, Poland

${ }^{3}$ Department of Thrombosis and Hemostasis, Leiden University Medical Center, Leiden, The Netherlands

${ }^{4}$ RTI Health Solutions, Research Triangle Institute, Research Triangle Park, North Carolina, United States

${ }^{5}$ Department of Medicine, Boehringer Ingelheim, Burlington, Canada

6 Department of Clinical Development and Medical Affairs, Boehringer Ingelheim Pharma GmbH \& Co KG, Ingelheim, Germany

7 Department of Neurology, University of Duisburg-Essen, Duisburg, Germany

${ }^{8}$ Clínica y Maternidad Suizo Argentina, Buenos Aires, Argentina

${ }^{9}$ Icahn School of Medicine at Mount Sinai, New York, New York, United States

10 Department of Cardiology, Atrial Fibrillation Center, Beijing Anzhen Hospital, Capital Medical University, Beijing, China

${ }^{11}$ Department of Cardiology, Atrial Fibrillation Center, Boehringer Ingelheim, Ingelheim, Germany

12 Department of Biostatistics and Data Sciences, Boehringer Ingelheim Pharma GmbH \& Co KG, Ingelheim, Germany

13 Department of Biostatistics and Data Sciences, Boehringer Ingelheim Pharmaceuticals, Inc., Ridgefield, Connecticut, United States

${ }^{14}$ Aalborg Thrombosis Research Unit, Department of Clinical Medicine, Aalborg University, Aalborg, Denmark
Address for correspondence Prof. Gregory Y.H. Lip, MD, Institute of Cardiovascular Sciences, University of Birmingham, City Hospital, Dudley Road, Birmingham B18 7QH, United Kingdom (e-mail: g.y.h.lip@bham.ac.uk).

Thromb Haemost 2017;117:2376-2388.
Abstract
Keywords
- atrial fibrillation
- anticoagulation
- stroke prevention
- regional differences
- GLORIA-AF

Introduction Although guideline-adherent antithrombotic therapy (ATT) for stroke prevention in atrial fibrillation (AF) is associated with lower mortality and thromboembolism, ATT uptake shows geographic variation worldwide. We aimed to assess thromboembolic risk and baseline ATT by geographic region and identify factors associated with prescription of ATT in a large, truly global registry of patients with recently diagnosed AF. Methods and Results Our analysis comprises 15,092 patients newly diagnosed with non-valvular AF at risk for stroke, enrolled in Phase II of Global Registry on Long-Term Oral Antithrombotic Treatment in Patients with Atrial Fibrillation (GLORIA-AF). Global oral anticoagulation (OAC) use was $79.9 \%$, being highest in Europe $(90.1 \%$ ), followed by

The review process for this paper was fully handled by Christian

Weber, Editor in Chief.

received

August 13, 2017

accepted

August 16, 2017
DOI https://doi.org/

10.1160/TH17-08-0555

ISSN 0340-6245.
Copyright (๑) 2017 Schattauer

License terms

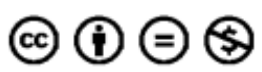


Africa/Middle East (87.4\%) and Latin America (85.3\%), North America (78.3\%) and Asia (55.2\%). Among OAC users, vitamin K antagonists (VKAs) have been replaced by nonVKA OACs (NOACs) as the more prevalent OAC option in all regions, with highest use in North America (66.5\%) and lowest in Asia (50.2\%). In Asia, OAC was $80.4 \%$ in community hospitals but only $49.8 \%$ in university hospitals and $42.6 \%$ in specialist offices, and varied from $21.0 \%$ in China to $89.7 \%$ in Japan (NOACs at $5.8 \%$ in China and $83.3 \%$ in Japan). Globally, $76.5 \%$ of low-risk patients were prescribed ATT (46.1\% OAC), whereas $17.7 \%$ high-risk patients were not anticoagulated (Europe $8.8 \%$; North America 18.9\%; Asia 42.4\%).

Conclusion Substantial inter- and intra-regional differences in ATT for stroke prevention in AF are evident in this global registry. While guideline-adherent ATT can be further improved, NOACs are the main contributor to high OAC use worldwide.

\section{Introduction}

Atrial fibrillation (AF) increases the risk of stroke and allcause mortality. ${ }^{1-3}$ Oral anticoagulation (OAC) with vitamin $\mathrm{K}$ antagonists (VKAs) such as warfarin reduces stroke risk by $64 \%$ and mortality by $26 \%$, compared with placebo/control, while aspirin decreases stroke risk by an estimated $19 \%$ and has no discernible effect on mortality. ${ }^{4}$ More recent clinical trials have demonstrated that non-vitamin $\mathrm{K}$ antagonist OACs (NOACs) are at least as effective as warfarin in stroke prevention and may offer greater safety and convenience. ${ }^{5-9}$

After identification of 'low-risk' patients (congestive heart failure, hypertension, age $\geq 75$ years [doubled], diabetes, stroke/transient ischaemic attack (TIA)/thromboembolism [doubled], vascular disease [prior myocardial infarction, peripheral arterial disease or aortic plaque], age 65-74 years, sex category [female] [ $\mathrm{CHA}_{2} \mathrm{DS}_{2}$-VASc] score 0 in males, 1 in females) who do not need any antithrombotic therapy, stroke prevention with OAC should generally be considered in patients with $\geq 1$ stroke risk factors, given the positive net clinical benefit when balancing reduced stroke risk against the risk of major bleeding. ${ }^{10}$ However, substantial geographic differences in use of antithrombotic therapy have been reported, ${ }^{11-13}$ and these differences almost certainly affect thromboembolic and mortality rates. ${ }^{14-16}$

The Global Registry on Long-Term Oral Antithrombotic Treatment in Patients with Atrial Fibrillation (GLORIA-AF) is one of the largest ongoing, international $\mathrm{AF}$ registries, which aims to assess safety, effectiveness and utilization patterns of antithrombotic therapy. ${ }^{17}$ The primary objective of this article is to explore regional differences in thromboembolic risk and treatment strategies in 15,092 AF patients from phase II of the registry. We also examined the magnitude of influence of baseline variables in regard to antithrombotic treatment choice.

\section{Methods}

\section{Study Design}

Details of GLORIA-AF study design have been previously published. ${ }^{17}$ In brief, GLORIA-AF is a prospective registry of patients with newly diagnosed AF at risk for stroke. The registry consists of three overlapping phases: phase I was the period before NOAC introduction, phase II began immediately following approval of the first NOAC in a given country and phase III started once propensity score comparisons indicated a substantial overlap in the range of the scores for those receiving dabigatran and those receiving VKA, to facilitate a valid assessment of safety and effectiveness. This report analyses baseline, cross-sectional data coming from patients enrolled in phase II of the registry (between November 2011 and December 2014).

\section{Patients and Settings}

The registry aims to enrol up to 56,000 AF patients from nearly 50 countries worldwide, from five geographical regions: (1) Asia, (2) Europe, (3) North America, (4) Latin America and (5) Africa/Middle East ( $\mathbf{- F i g . 1}$ ). The programme includes up to 2,200 sites in a variety of in- and outpatient settings, and involves various medical specialties. Inclusion criteria were as follows: adult patients, new onset $(<3$ months before study entry, with the exception Latin America, where it was $<4.5$ months due to referral patterns) non-valvular $\mathrm{AF}$, and $\geq 1$ risk factor for stroke as per the $\mathrm{CHA}_{2} \mathrm{DS}_{2}$ VASc score. Main exclusion criteria were as follows: mechanical heart valves or valve disease requiring surgical valve replacement, history of OAC with VKA less than 60 days for any indication, generally reversible cause of AF, an indication other than AF for VKA treatment and life expectancy less than 1 year.

Thromboembolic risk was assessed with the use of $\mathrm{CHA}_{2} \mathrm{DS}_{2}$-VASc score. ${ }^{18}$ 'Low-risk' patients were defined as $\mathrm{CHA}_{2} \mathrm{DS}_{2}$-VASc $=1$ in females (males with $\mathrm{CHA}_{2} \mathrm{DS}_{2}$-VASc $=0$ were not included); 'moderate-risk' patients as males with $\mathrm{CHA}_{2} \mathrm{DS}_{2}$-VASc $=1$; and 'high-risk' patients as those with $\mathrm{CHA}_{2} \mathrm{DS}_{2}$-VASc score $\geq 2$. Bleeding risk assessment was based on the HAS-BLED (hypertension, abnormal renal and liver function, stroke, bleeding, labile INR [international normalized ratio] values, age $\geq 65$ years, drugs or alcohol) score, and high-risk was defined as HAS-BLED $\geq 3$. $^{19}$

\section{Data Collection}

An Electronic Data Capture System (Cambridge, Massachusetts, United States) was used to collect and store data as well as to ensure safety and confidentiality. High level of data integrity is ensured by in-person monitoring, bimonthly calls to all sites 


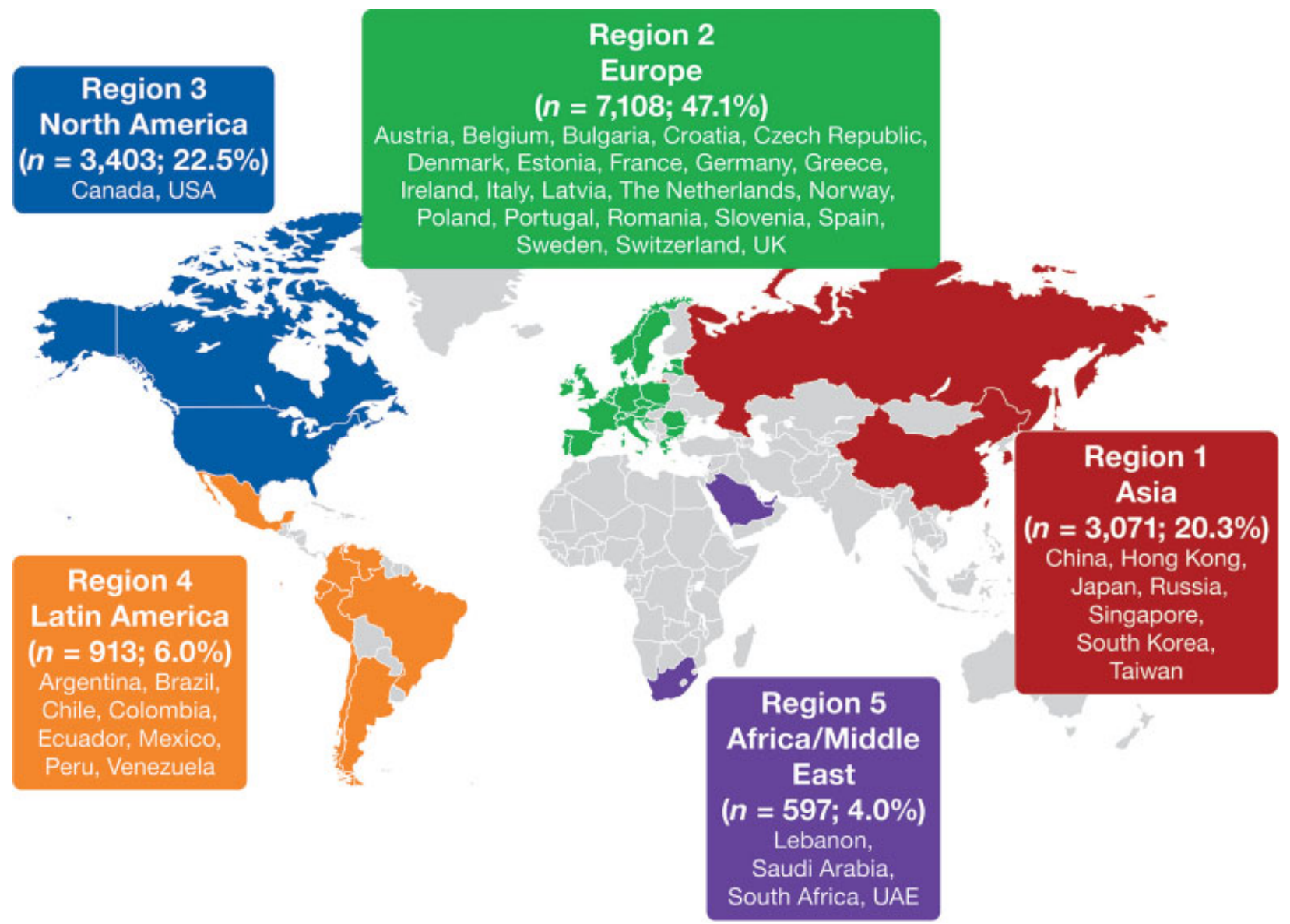

Fig. 1 Definition of geographical regions and country participation in Global Registry on Long-Term Oral Antithrombotic Treatment in Patients with Atrial Fibrillation (GLORIA-AF).

to review data quality, entry and open queries, biweekly updates of follow-up on data trends (e.g., missing data, termination rates, overdue forms, vital status), regular visits by audit teams as well as quarterly medical review meetings to assess aggregate data. Local investigators were instructed in detail regarding system requirements and functionality as well as encouraged to enrol consecutive eligible AF patients. All patients entered the study after written, informed consent, and the study complies with the Declaration of Helsinki.

\section{Statistical Analysis}

Baseline data were summarized descriptively. Continuous variables were reported as median and quartiles. Categorical variables were reported as absolute frequencies and percentages.

Logistic regression provides odds ratios (OR), which only closely approximate risk ratios (i.e., relative risks) for rare outcomes $(<10 \%)$. Because use of OAC and NOAC was prevalent, we used log-binomial regression to estimate the risk ratios directly. ${ }^{20}$ From here on and throughout the text, we will use the term 'probability ratio' rather than 'risk ratio' as our measure describes drug use rather than adverse outcomes.

Both univariate and multivariable log-binomial regression analyses were conducted to, respectively, evaluate the crude and adjusted probability ratios (together with 95\% confidence intervals [CIs]) of OAC among all eligible patients, and of NOAC treatment among patients receiving OAC, associated with the variables of clinical relevance: age, gender, type of $\mathrm{AF}$, categorization of $\mathrm{AF}, \mathrm{AF}$ cardioversion, hypertension, diabetes mellitus, previous stroke/TIA, congestive heart failure, coronary artery disease, myocardial infarction, peripheral artery disease, previous bleeding events, chronic kidney disease, alcohol abuse, cancer, chronic gastrointestinal disease, $\mathrm{CHADS}_{2}$ score, $\mathrm{CHA}_{2} \mathrm{DS}_{2}$-VASc score, HAS-BLED score, geographic region, type of site and medical treatment reimbursement. No variable selection procedure was applied in the multivariable analyses. The COPY method was used to obtain approximate maximum likelihood estimates where the log-binomial model does not converge. ${ }^{21}$ All analyses were performed using SAS statistical software version 9.4 (SAS Institute, Inc., Cary, North Carolina, United States). Refer to online supplementary materials for more details on methodology and statistical analysis.

\section{Results}

\section{Baseline Data}

Baseline characteristics by region are summarized in - Table 1 . Patients from Asia were younger, with $28.5 \%$ being $\geq 75$ years, compared with Europe (44.2\%) and North America (39.6\%). The most prevalent comorbidities were hypertension (varying 
Table 1 Baseline characteristics by region

\begin{tabular}{|c|c|c|c|c|c|c|}
\hline$N(\%)$ or median [Q1,Q3] & $\begin{array}{l}\text { All } \\
n=15,092 \\
(100 \%)\end{array}$ & $\begin{array}{l}\text { Region } 1 \\
\text { Asia, } n=3,071 \\
(20.3 \%)\end{array}$ & $\begin{array}{l}\text { Region } 2 \\
\text { Europe, } n=7,108 \\
(47.1 \%)\end{array}$ & $\begin{array}{l}\text { Region } 3 \\
\text { North America, } \\
n=3,403(22.5 \%)\end{array}$ & $\begin{array}{l}\text { Region } 4 \\
\text { Latin America, } \\
n=913(6.0 \%)\end{array}$ & $\begin{array}{l}\text { Region } 5 \\
\text { Africa/Middle East, } \\
n=597(4.0 \%)\end{array}$ \\
\hline \multicolumn{7}{|l|}{ Patient characteristics } \\
\hline Age, y, median [Q1,Q3] & $71.0[64-78]$ & $68.0[60-76]$ & $73.0[66-79]$ & $71.0[64-79]$ & $71.0[62-78]$ & $70.0[61-76]$ \\
\hline Age, $\geq 75 \mathrm{y}$ & $5,907(39.1)$ & $874(28.5)$ & $3,144(44.2)$ & $1,349(39.6)$ & $354(38.8)$ & $186(31.2)$ \\
\hline Female gender & $6,872(45.5)$ & $1,337(43.5)$ & $3,317(46.7)$ & $1,524(44.8)$ & $410(44.9)$ & $284(47.6)$ \\
\hline GFR, mL/min/1.73 m², median [Q1,Q3] & 75.1 [61-90] & 78.7 [65-96] & $74.0[61-88]$ & 74.3 [59-89] & $72.5[59-87]$ & 75.2 [58-92] \\
\hline No reimbursement for medications & $1,076(7.1)$ & $375(12.2)$ & $313(4.4)$ & $103(3.0)$ & $183(20.0)$ & $102(17.1)$ \\
\hline \multicolumn{7}{|l|}{ Medical history } \\
\hline Hypertension & $11,255(74.6)$ & $2,130(69.4)$ & $5,217(73.4)$ & $2,738(80.5)$ & $690(75.6)$ & $480(80.4)$ \\
\hline Hyperlipidaemia & $6,026(39.9)$ & $821(26.7)$ & $2,595(36.5)$ & $2,085(61.3)$ & $246(26.9)$ & $279(46.7)$ \\
\hline Diabetes mellitus & $3,487(23.1)$ & $619(20.2)$ & $1,510(21.2)$ & $921(27.1)$ & $185(20.3)$ & $252(42.2)$ \\
\hline Previous stroke/TIA & $2,147(14.2)$ & $400(13.0)$ & $1,107(15.6)$ & $422(12.4)$ & $110(12.0)$ & $108(18.1)$ \\
\hline Congestive heart failure & $3,647(24.2)$ & $837(27.3)$ & $1,662(23.4)$ & $671(19.7)$ & $296(32.4)$ & $181(30.3)$ \\
\hline Coronary artery disease & $3,068(20.3)$ & $674(21.9)$ & $1,167(16.4)$ & $919(27.0)$ & $114(12.5)$ & $194(32.5)$ \\
\hline Myocardial infarction & $1,600(10.6)$ & $246(8.0)$ & $741(10.4)$ & $418(12.3)$ & $96(10.5)$ & $99(16.6)$ \\
\hline Abnormal kidney function ${ }^{a}$ & $241(1.6)$ & $45(1.5)$ & $89(1.3)$ & $75(2.2)$ & $12(1.3)$ & $20(3.4)$ \\
\hline \multicolumn{7}{|l|}{ Type of AF } \\
\hline Paroxysmal & $8,052(53.4)$ & $1,804(58.7)$ & $3,326(46.8)$ & $2,240(65.8)$ & $396(43.4)$ & $286(47.9)$ \\
\hline Persistent & $5,362(35.5)$ & $1,086(35.4)$ & $2,763(38.9)$ & $1,007(29.6)$ & $316(34.6)$ & $190(31.8)$ \\
\hline Permanent & $1,678(11.1)$ & $181(5.9)$ & $1,019(14.3)$ & $156(4.6)$ & $201(22.0)$ & $121(20.3)$ \\
\hline \multicolumn{7}{|l|}{ Categorization of AF } \\
\hline Symptomatic & $4,263(28.2)$ & $751(24.5)$ & $2,258(31.8)$ & $804(23.6)$ & $292(32.0)$ & $158(26.5)$ \\
\hline Minimally symptomatic & $6,004(39.8)$ & $1,460(47.5)$ & $2,671(37.6)$ & $1,278(37.6)$ & $323(35.4)$ & $272(45.6)$ \\
\hline Asymptomatic & $4,825(32.0)$ & $860(28.0)$ & $2,179(30.7)$ & $1,321(38.8)$ & $298(32.6)$ & $167(28.0)$ \\
\hline \multicolumn{7}{|l|}{ Interventions in AF } \\
\hline AF cardioversion & $2,431(16.1)$ & $331(10.8)$ & $1,226(17.2)$ & $646(19.0)$ & $143(15.7)$ & $85(14.2)$ \\
\hline AF ablation & $161(1.1)$ & $93(3.0)$ & $33(0.5)$ & $26(0.8)$ & $5(0.5)$ & $4(0.7)$ \\
\hline $\mathrm{CHA}_{2} \mathrm{DS}_{2}$-VASC & 3.0 & 3.0 & 3.0 & 3.0 & 3.0 & 3.0 \\
\hline Low risk (score 1 in women) & $332(2.2)$ & $110(3.6)$ & $114(1.6)$ & $83(2.4)$ & $14(1.5)$ & $11(1.8)$ \\
\hline Moderate risk (score 1 in men) & $1,761(11.7)$ & $532(17.3)$ & $684(9.6)$ & $387(11.4)$ & $108(11.8)$ & $50(8.4)$ \\
\hline High risk (score $\geq 2$ ) & $12,999(86.1)$ & $2,429(79.1)$ & $6,310(88.8)$ & $2,933(86.2)$ & $791(86.6)$ & $536(89.8)$ \\
\hline HAS-BLED & 1.0 & 1.0 & 1.0 & 1.0 & 1.0 & 1.0 \\
\hline Low risk (score $0-2$ ) & $11,927(79.0)$ & $2,359(76.8)$ & $5,538(77.9)$ & $2,775(81.5)$ & $772(84.6)$ & $483(80.9)$ \\
\hline High risk (score $\geq 3$ ) & $1,376(9.1)$ & $329(10.7)$ & $575(8.1)$ & $352(10.3)$ & $59(6.5)$ & $61(10.2)$ \\
\hline Missing & 1,789 (11.9) & $383(12.5)$ & $995(14.0)$ & $276(8.1)$ & $82(9.0)$ & $53(8.9)$ \\
\hline \multicolumn{7}{|l|}{ Physician specialty } \\
\hline Cardiologist & $13,863(91.9)$ & 2,994 (97.5) & $6,568(92.4)$ & $2,872(84.4)$ & 864 (94.6) & 565 (94.6) \\
\hline GP/geriatrician & $359(2.4)$ & $34(1.1)$ & $63(0.9)$ & $229(6.7)$ & $32(3.5)$ & $1(0.2)$ \\
\hline Internist & $322(2.1)$ & $12(0.4)$ & $71(1.0)$ & $197(5.8)$ & $17(1.9)$ & $25(4.2)$ \\
\hline Neurologist & $147(1.0)$ & $7(0.2)$ & $63(0.9)$ & $77(2.3)$ & $0(0.0)$ & $0(0.0)$ \\
\hline Other & $386(2.6)$ & $24(0.8)$ & $328(4.6)$ & $28(0.8)$ & $0(0.0)$ & $6(1.0)$ \\
\hline Missing & $15(0.1)$ & $0(0.0)$ & $15(0.2)$ & $0(0.0)$ & $0(0.0)$ & $0(0.0)$ \\
\hline \multicolumn{7}{|l|}{ Type of setting } \\
\hline GP/primary care & $968(6.4)$ & $178(5.8)$ & $216(3.0)$ & $496(14.6)$ & $75(8.2)$ & $3(0.5)$ \\
\hline Specialist office & $4,567(30.3)$ & $611(19.9)$ & $1,200(16.9)$ & $2,290(67.3)$ & $320(35.0)$ & $146(24.5)$ \\
\hline Community hospital & $3,969(26.3)$ & $561(18.3)$ & 2,717 (38.2) & $298(8.8)$ & $251(27.5)$ & $142(23.8)$ \\
\hline University hospital & $5,081(33.7)$ & $1,659(54.0)$ & $2,715(38.2)$ & $265(7.8)$ & $207(22.7)$ & $235(39.4)$ \\
\hline Outpatient/anticoagulation clinic & $381(2.5)$ & $62(2.0)$ & $143(2.0)$ & $54(1.6)$ & $51(5.6)$ & $71(11.9)$ \\
\hline Other & $126(0.8)$ & $0(0.0)$ & $117(1.6)$ & $0(0.0)$ & $9(1.0)$ & $0(0.0)$ \\
\hline
\end{tabular}

Abbreviations: AF, atrial fibrillation; $\mathrm{CHA}_{2} \mathrm{DS}_{2}$-VASc, congestive heart failure, hypertension, age $\geq 75$ years (doubled), diabetes, stroke/transient ischaemic attack/thromboembolism (doubled), vascular disease (prior myocardial infarction [MI], peripheral arterial disease [PAD] or aortic plaque), age 65 to 74 years, sex category (female); GFR, glomerular filtration rate; GP, general practitioner; HAS-BLED, hypertension, abnormal renal and liver function, stroke, bleeding, labile INR (international normalized ratio), age $\geq 65$ years, drugs or alcohol; TIA, transient ischaemic attack.

${ }^{a}$ Defined as the presence of chronic dialysis or renal transplantation or serum creatinine $\geq 200 \mu \mathrm{mol} / \mathrm{L}$. 
from $80.5 \%$ in North America to $69.4 \%$ in Asia) and hyperlipidaemia (61.3\% in North America, 26.7\% in Asia). Diabetes was most common in Africa/Middle East (42.2\%) and less frequent in Asia (20.2\%). The proportion of secondary prevention patients (with prior stroke/TIA), as well as those with coronary artery disease and previous myocardial infarction, was highest in Africa/Middle East (18.1, 32.5 and 16.6\%, respectively). Congestive heart failure was most common in Latin America (32.4\%) and lowest in North America (19.7\%).

The vast majority of patients were treated by cardiologists (91.9\%), with some differences among regions (97.5\% in Asia and $84.4 \%$ in North America). In Asia, 54\% of patients were enrolled in university hospitals, while in North America most of the subjects were recruited in specialist offices (67.3\%).

Overall, $79.9 \%$ of all patients were prescribed OAC: $32.3 \%$ VKA and $47.6 \%$ NOACs (dabigatran $31.6 \%$, rivaroxaban $11.4 \%$ and apixaban $4.6 \%$ ), while $12.1 \%$ received antiplatelet drugs (aspirin in $93.1 \%$ of cases) and $7.8 \%$ received no antithrombotic therapy. Of those at low bleeding risk, $81.8 \%$ were anticoagulated, $9.6 \%$ received aspirin, $0.6 \%$ other antithrombotic drugs and $8.1 \%$ were not treated. The corresponding proportions for those at high risk of bleeding (HAS-BLED $\geq 3$ ) were $63.4,27.6,3.6$ and $5.3 \%$.

\section{Antithrombotic Therapy by Region}

The highest OAC rates were observed in Europe (90.1\%), followed by Africa/Middle East (87.4\%), Latin America (85.3\%) and North America (78.3\%), while in Asia it was $55.2 \%$ (-Fig. 2A). In the subgroup of patients on OACs ( $n=12,065 ; 79.9 \%$ ), the most prevalent option was NOACs in North America (66.5\%), Latin America (66.0\%) and Africa/ Middle East (63.6\%; - Fig. 2B). In Asia, the probability that a patient with AF remained untreated was the highest, at $19.8 \%$, and in the Africa/Middle East region, it was the lowest, at 1.5\%. Antiplatelet therapy was common in Asia (25.0\%) and least prevalent in Europe (6.0\%; - Fig. 2A).

A Antithrombotic treatment by region (entire population)

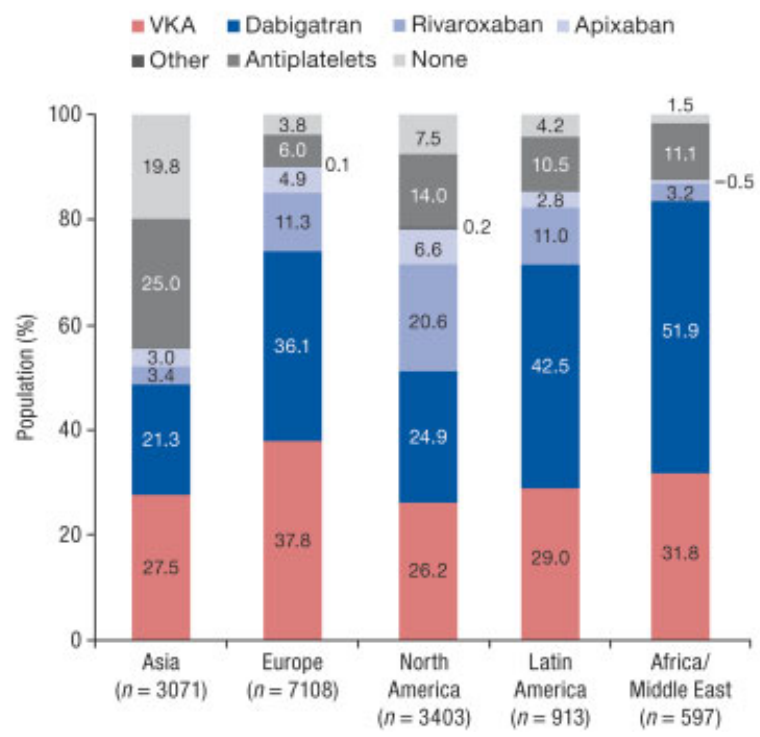

\section{Antithrombotic Therapy by Region and Stroke Risk}

In Europe, $64.9 \%$ of low-risk patients were prescribed OAC and 14.9\% antiplatelets. In North America, the corresponding numbers were 39.8 and $49.4 \%$, respectively, while in Asia, 28.2 and $32.7 \%$, respectively (-Fig. 3). Among European patients at moderate risk of stroke, $85.1 \%$ received OAC, compared with 79.6\% in Latin America and 74.0\% in Africa/Middle East. Corresponding proportions in North America and Asia were 67.7 and $49.4 \%$, respectively. In high-risk patients, OAC use was highest in Europe (91.1\%), followed by Africa/Middle East (89.2\%), Latin America (86.6\%), North America (80.8\%) and Asia (57.6\%).

\section{Antithrombotic Therapy by Region and Country}

- Table 2 summarizes enrolment rate by regions and countries with their respective antithrombotic treatment patterns. Europe was the highest recruiting region with 7,108 patients (47.1\%) enrolled, whereas United States was the highest recruiting country ( $n=3,007 ; 19.9 \%$ ). The greatest heterogeneity in OAC prescription was noted in Asia, where anticoagulation varied from $21.0 \%$ in China to $89.7 \%$ in Japan. For Europe, generally high overall OAC rates were observed in all countries, and with the exception of only a few high-enrolling countries ( $>500$ patients enrolled, i.e., the Netherlands, Spain, United Kingdom), NOACs were the more prevalent OAC option.

\section{Antithrombotic Therapy by Region and Clinical Setting} In Asia, $80.4 \%$ of patients from community hospitals were anticoagulated, compared with 49.8 and $42.6 \%$ of those from university hospitals and specialist offices, respectively (-Fig. 4). NOACs and VKAs were prescribed for 71.1 and $9.3 \%$ of patients from community hospitals; in university hospitals, the corresponding numbers were 11.0 and $38.8 \%$, and in specialist offices, 21.6 and $20.9 \%$, respectively.

Overall, physicians from university hospitals prescribed more VKAs (42.7\%) and less NOACs (33.0\%). In North America, the highest anticoagulation use was reported by university

B Oral anticoagulation by region (OAC users only)

=VKA $=$ Dabigatran $=$ Rivaroxaban $=$ Apixaban

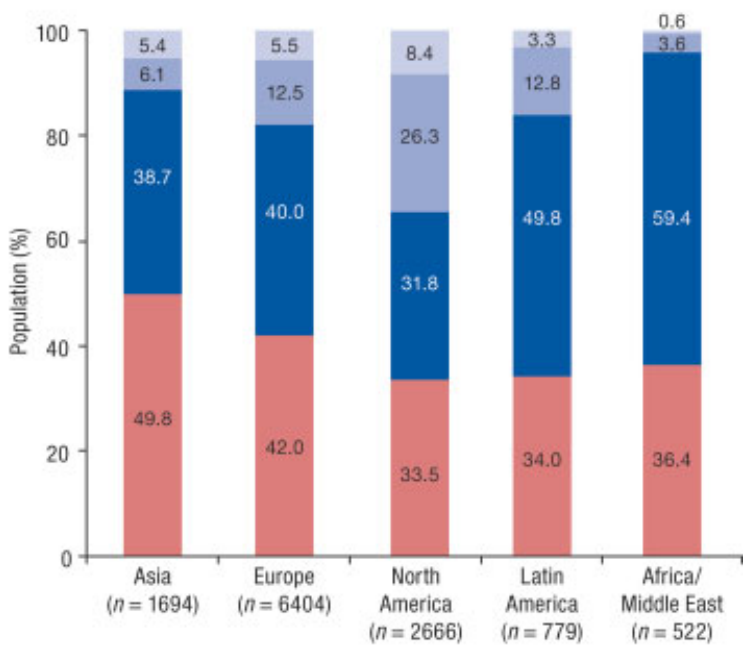

Fig. 2 Antithrombotic treatments by region, overall (A) and among oral anticoagulant (OAC) users (B). Other antithrombotic therapy includes the combination of antithrombotic agents. 

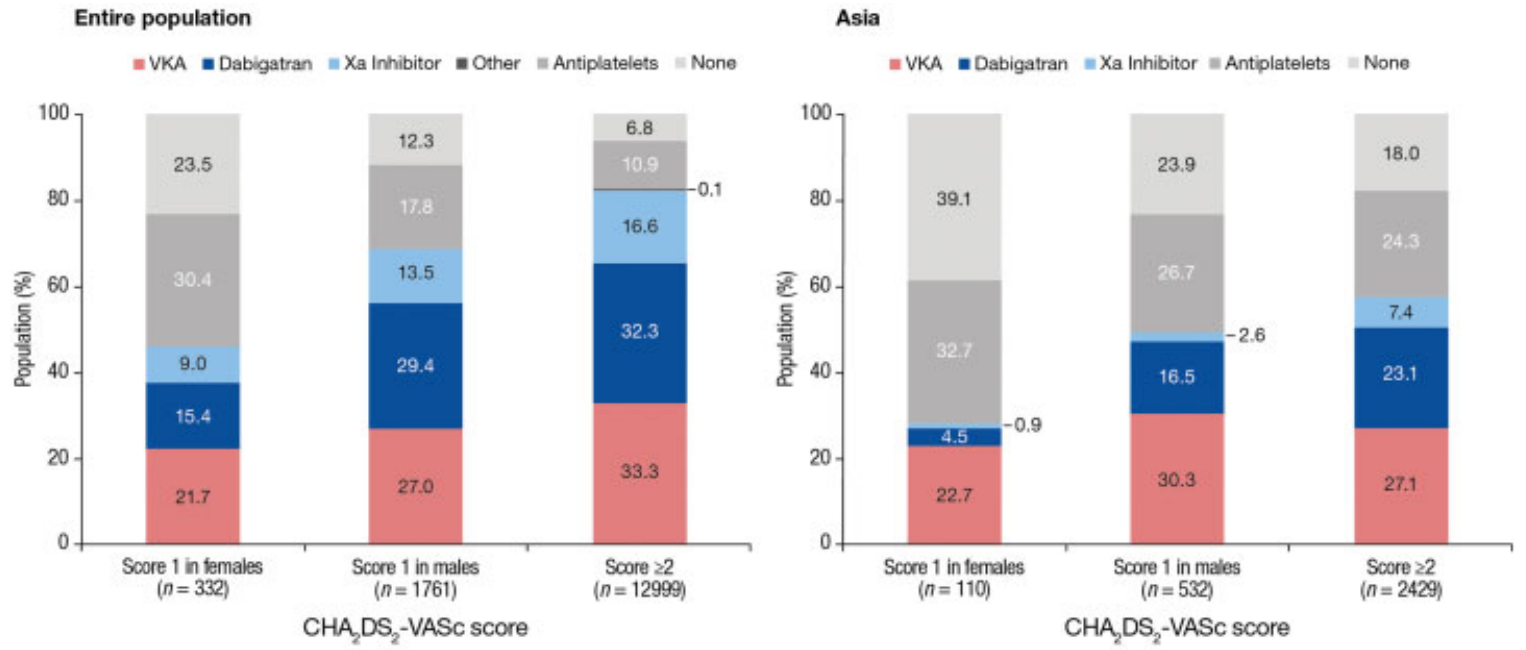

\section{Europe}

\section{North America}

$=$ VKA $=$ Dabigatran $=$ Xa Inhibitor $=$ Other $=$ Antiplatelets $=$ None
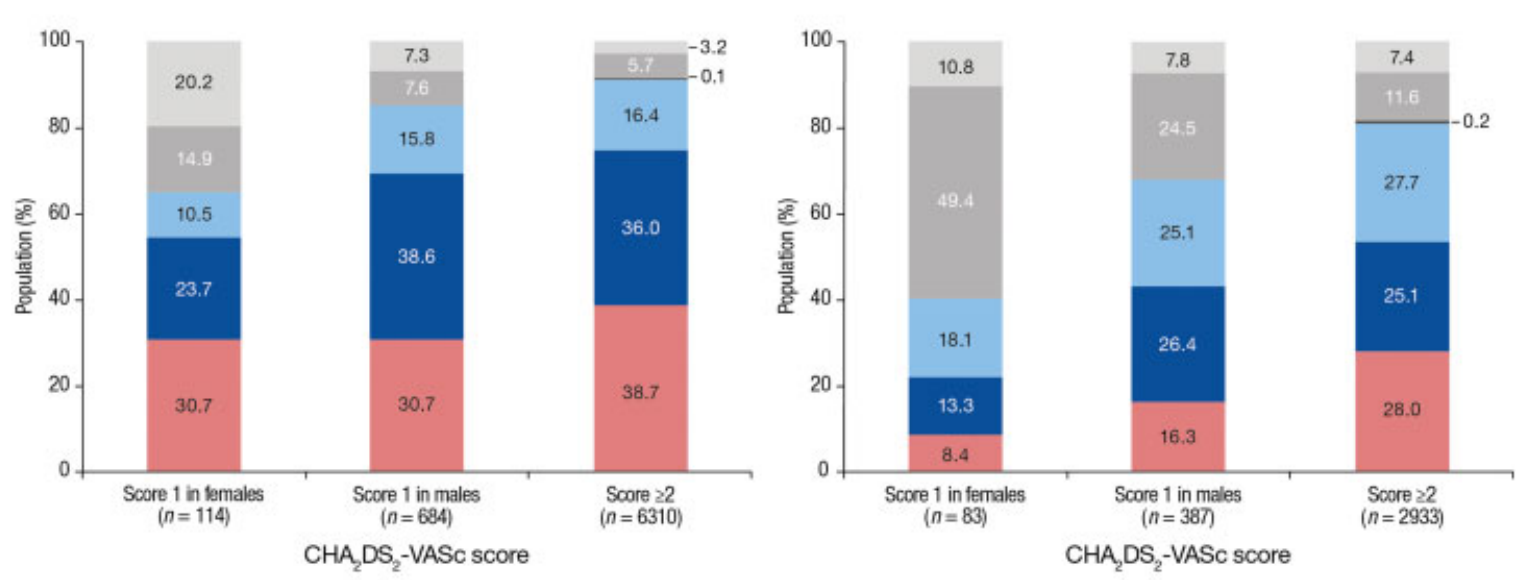

Latin America

Africa/Middle East

$=$ VKA $=$ Dabigatran $=$ Xa Inhibitor $=$ Antiplatelets $=$ None

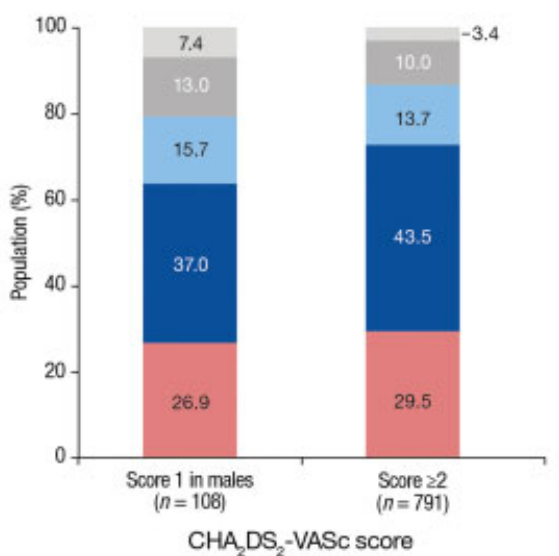

=VKA $=$ Dabigatran $=$ Xa Inhibitor $=$ Antiplatelets $=$ None

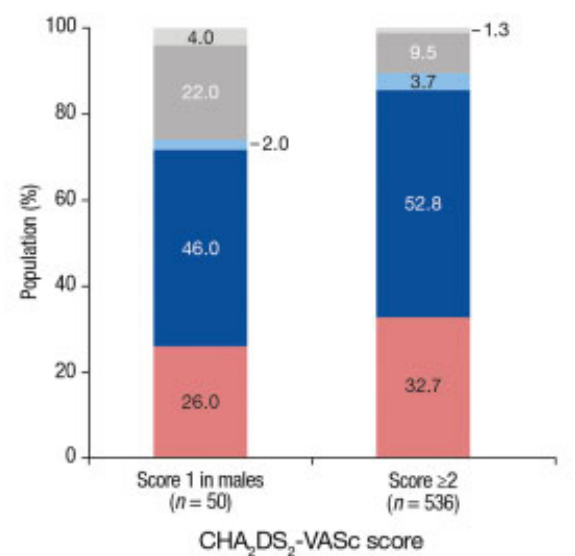

Fig. 3 Antithrombotic treatment by region and stroke risk. ${ }^{\text {a }}$ Other = antiplatelets other than acetylsalicylic acid (ASA) and combination of antithrombotic agents; Xa inhibitor = rivaroxaban, apixaban. ${ }^{\text {II }}$ Latin America and Africa/Middle East, score $=1$ in females is not shown as there were too low patient numbers to calculate meaningful percentages for comparison.

hospitals at $83.0 \%$ (53.6\% NOACs and 29.4\% VKAs), compared with $69.8 \%$ in community hospitals and $70.6 \%$ in general practices. The highest prevalence of aspirin treatment or non-treatment was seen not only in Asian specialist offices (55.8\%) and university hospitals (48.5\%), but also in American community hospitals (29.5\%) and general practices (29.0\%).

\section{Anticoagulation Therapy versus Other Treatment}

A log-binomial regression analysis for estimation of probability ratios for the prescription of anticoagulation therapy (VKA, NOAC) versus other treatment (antiplatelet or no treatment) is presented in -Supplementary Table S1 (online only). In the multivariable analysis, adjusted 
Table 2 Antithrombotic therapy by region and country

\begin{tabular}{|c|c|c|c|c|c|c|c|}
\hline Entire population & $\begin{array}{l}\text { All }^{\mathrm{a}} \\
15,092(100)\end{array}$ & $\begin{array}{l}\text { None } \\
1,182(7.8)\end{array}$ & $\begin{array}{l}\text { ASA } \\
1,706(11.3)\end{array}$ & $\begin{array}{l}\text { VKA } \\
4,878(32.3)\end{array}$ & $\begin{array}{l}\text { NOAC } \\
7,187(47.6)\end{array}$ & $\begin{array}{l}\text { Overall OAC } \\
12,065(79.9)\end{array}$ & $\begin{array}{l}\text { Other } \\
139(0.9)\end{array}$ \\
\hline Asia & $3,071(100)$ & 608 (19.8) & $727(23.7)$ & $844(27.5)$ & $850(27.7)$ & $1,694(55.2)$ & $42(1.4)$ \\
\hline China & $1,018(33.1)$ & $431(42.3)$ & $351(34.5)$ & $155(15.2)$ & $59(5.8)$ & $214(21.0)$ & $22(2.2)$ \\
\hline Hong Kong & $49(1.6)$ & $3(6.1)$ & $13(26.5)$ & $9(18.4)$ & $24(49.0)$ & $33(67.3)$ & 0 \\
\hline Japan & $312(10.2)$ & $27(8.7)$ & $5(1.6)$ & $20(6.4)$ & $260(83.3)$ & $280(89.7)$ & 0 \\
\hline Russian Federation & $404(13.2)$ & $2(0.5)$ & $45(11.1)$ & $75(18.6)$ & $282(69.8)$ & $357(88.4)$ & 0 \\
\hline Singapore & $48(1.6)$ & $5(10.4)$ & $5(10.4)$ & $23(47.9)$ & $14(29.2)$ & $37(77.1)$ & $1(2.1)$ \\
\hline South Korea & $997(32.5)$ & $117(11.7)$ & $282(28.3)$ & $541(54.3)$ & $42(4.2)$ & $583(58.5)$ & $15(1.5)$ \\
\hline Taiwan & $243(7.9)$ & $23(9.5)$ & $26(10.7)$ & $21(8.6)$ & 169 (69.5) & $190(78.2)$ & $4(1.6)$ \\
\hline Europe & $7,108(100)$ & $272(3.8)$ & $373(5.2)$ & $2,687(37.8)$ & $3,717(52.3)$ & $6,404(90.1)$ & $59(0.8)$ \\
\hline Austria & $62(0.9)$ & $1(1.6)$ & 0 & $5(8.1)$ & $56(90.3)$ & $61(98.4)$ & 0 \\
\hline Belgium & $38(0.5)$ & $4(10.5)$ & $2(5.3)$ & $3(8.0)$ & $29(76.3)$ & $32(84.2)$ & 0 \\
\hline Bulgaria & $303(4.3)$ & $3(1.0)$ & $6(2.0)$ & $61(20.1)$ & $233(76.9)$ & $294(97.0)$ & 0 \\
\hline Croatia & $165(2.3)$ & $11(6.7)$ & $36(21.8)$ & $96(58.2)$ & $22(13.3)$ & $118(71.5)$ & 0 \\
\hline Czech Republic & $75(1.1)$ & $7(9.3)$ & $3(4.0)$ & $53(70.7)$ & $12(16.0)$ & $65(86.7)$ & 0 \\
\hline Denmark & $62(0.9)$ & $2(3.2)$ & 0 & $14(22.6)$ & $46(74.2)$ & $60(96.8)$ & 0 \\
\hline Estonia & $45(0.6)$ & 0 & 0 & $17(37.8)$ & $28(62.2)$ & $45(100)$ & 0 \\
\hline France & 969 (13.6) & $21(2.2)$ & $22(2.3)$ & $195(20.1)$ & $729(75.2)$ & 924 (95.4) & $2(0.2)$ \\
\hline Germany & $1,220(17.2)$ & $39(3.2)$ & $75(6.1)$ & $201(16.5)$ & 899 (73.7) & $1,100(90.2)$ & $6(0.5)$ \\
\hline Greece & $270(3.8)$ & $7(2.6)$ & $9(3.3)$ & $113(41.9)$ & $141(52.2)$ & 254 (94.1) & 0 \\
\hline Republic of Ireland & $61(0.9)$ & $1(1.6)$ & $5(8.2)$ & $13(21.3)$ & $42(68.9)$ & $55(90.2)$ & 0 \\
\hline Italy & $359(5.1)$ & $17(4.7)$ & $14(3.9)$ & $111(30.9)$ & $214(59.6)$ & $325(90.5)$ & $3(0.8)$ \\
\hline Latvia & $60(0.8)$ & 0 & $20(33.3)$ & $6(10.0)$ & $34(56.7)$ & $40(66.7)$ & 0 \\
\hline The Netherlands & $528(7.4)$ & $9(1.7)$ & $12(2.3)$ & $317(60.0)$ & 188 (35.6) & 505 (95.6) & $2(0.4)$ \\
\hline Norway & $77(1.1)$ & $2(2.6)$ & $2(2.6)$ & $16(20.8)$ & $57(74.0)$ & $73(94.8)$ & 0 \\
\hline Poland & $75(1.1)$ & $4(5.3)$ & $3(4.0)$ & $9(12.0)$ & $59(78.7)$ & $68(90.7)$ & 0 \\
\hline Portugal & $164(2.3)$ & $5(3.0)$ & $3(1.8)$ & $56(34.1)$ & $100(61.0)$ & $156(95.1)$ & 0 \\
\hline Romania & $301(4.2)$ & $1(0.3)$ & $17(5.6)$ & $121(40.2)$ & $152(50.5)$ & $273(90.7)$ & $10(3.3)$ \\
\hline Slovenia & $68(1.0)$ & 0 & 0 & $2(2.9)$ & $66(97.1)$ & $68(100)$ & 0 \\
\hline Spain & $1,155(16.2)$ & $78(6.8)$ & $70(6.1)$ & $725(62.8)$ & $273(23.6)$ & $998(86.4)$ & $9(0.8)$ \\
\hline Sweden & $137(1.9)$ & 0 & 0 & $80(58.4)$ & $57(41.6)$ & $137(100)$ & 0 \\
\hline Switzerland & $72(1.0)$ & $1(1.4)$ & $3(4.2)$ & $20(27.8)$ & $47(65.3)$ & $67(93.1)$ & $1(1.4)$ \\
\hline United Kingdom & $842(11.8)$ & $59(7.0)$ & $71(8.4)$ & $453(53.8)$ & $233(27.7)$ & $686(81.5)$ & $26(3.1)$ \\
\hline North America & $3,403(100.0)$ & $255(7.5)$ & $455(13.4)$ & $892(26.2)$ & $1,774(52.1)$ & $2,666(78.3)$ & $27(0.8)$ \\
\hline Canada & 396 (11.6) & $8(2.0)$ & $47(11.7)$ & $101(25.5)$ & $238(60.1)$ & $339(85.6)$ & $2(0.5)$ \\
\hline United States & $3,007(88.4)$ & $247(8.2)$ & 408 (13.6) & $791(26.3)$ & $1,536(51.1)$ & $2,327(77.4)$ & $25(0.8)$ \\
\hline Latin America & $913(100.0)$ & $38(4.2)$ & $92(10.1)$ & $265(29.0)$ & $514(56.3)$ & $779(85.3)$ & $4(0.4)$ \\
\hline Argentina & $153(16.8)$ & $4(2.6)$ & $14(9.2)$ & $62(40.5)$ & $73(47.7)$ & $135(88.2)$ & 0 \\
\hline Brazil & 306 (33.5) & $28(9.2)$ & $42(13.7)$ & $99(32.4)$ & $137(44.8)$ & $236(77.1)$ & 0 \\
\hline Chile & $11(1.2)$ & 0 & $1(9.1)$ & $7(63.6)$ & $3(27.2)$ & $10(90.9)$ & 0 \\
\hline Colombia & $134(14.7)$ & $2(1.5)$ & $6(4.5)$ & $29(21.6)$ & $97(72.4)$ & $126(94.0)$ & 0 \\
\hline Ecuador & $36(4.0)$ & $1(2.8)$ & $5(13.9)$ & $5(13.9)$ & $23(63.9)$ & $28(77.8)$ & $2(5.6)$ \\
\hline Mexico & $220(24.1)$ & $1(0.5)$ & $21(9.5)$ & $47(21.4)$ & $149(67.7)$ & $196(89.1)$ & $2(0.9)$ \\
\hline Peru & $44(4.8)$ & $1(2.3)$ & $2(4.5)$ & $15(34.1)$ & $26(59.1)$ & $41(93.2)$ & 0 \\
\hline Venezuela & $9(1.0)$ & $1(11.1)$ & $1(11.1)$ & $1(11.1)$ & $6(66.7)$ & $7(77.8)$ & 0 \\
\hline Africa/Middle East & $597(100.0)$ & $9(1.5)$ & $59(9.9)$ & $190(31.8)$ & $332(55.6)$ & $522(87.4)$ & $7(1.2)$ \\
\hline Lebanon & $243(40.7)$ & $1(0.4)$ & $29(11.9)$ & $62(25.5)$ & $148(60.9)$ & $210(86.4)$ & $3(1.2)$ \\
\hline Saudi Arabia & $235(39.4)$ & $4(1.7)$ & $8(3.4)$ & $77(32.8)$ & $145(61.7)$ & $222(94.5)$ & $1(0.4)$ \\
\hline Republic of South Africa & $32(5.4)$ & $2(6.3)$ & $1(3.1)$ & $25(78.1)$ & $3(9.4)$ & $28(87.5)$ & $1(3.1)$ \\
\hline United Arab Emirates & $87(14.6)$ & $2(2.3)$ & $21(24.1)$ & $26(29.9)$ & $36(41.4)$ & $62(71.3)$ & $2(2.3)$ \\
\hline
\end{tabular}

Abbreviations: ASA, aspirin; NOAC, non-vitamin K antagonists oral anticoagulant; VKA, vitamin K antagonists; OAC, oral anticoagulant; Other, antiplatelets other than aspirin and combination of antithrombotic agents.

Note: Data are presented as $n(\%)-$ row percentages.

${ }^{\mathrm{a}}$ Data presented in column percentages. 

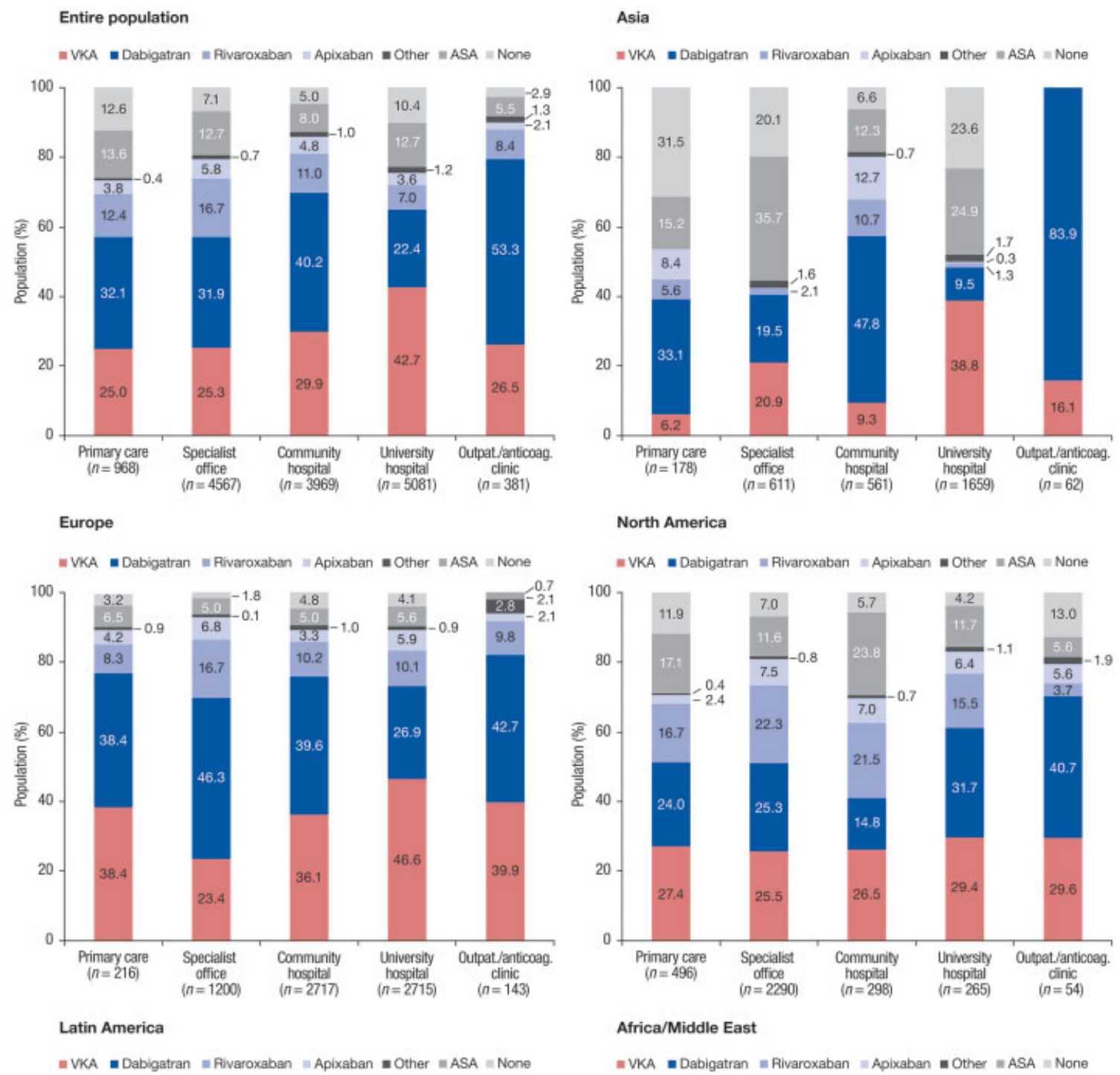

Africa/Middle East
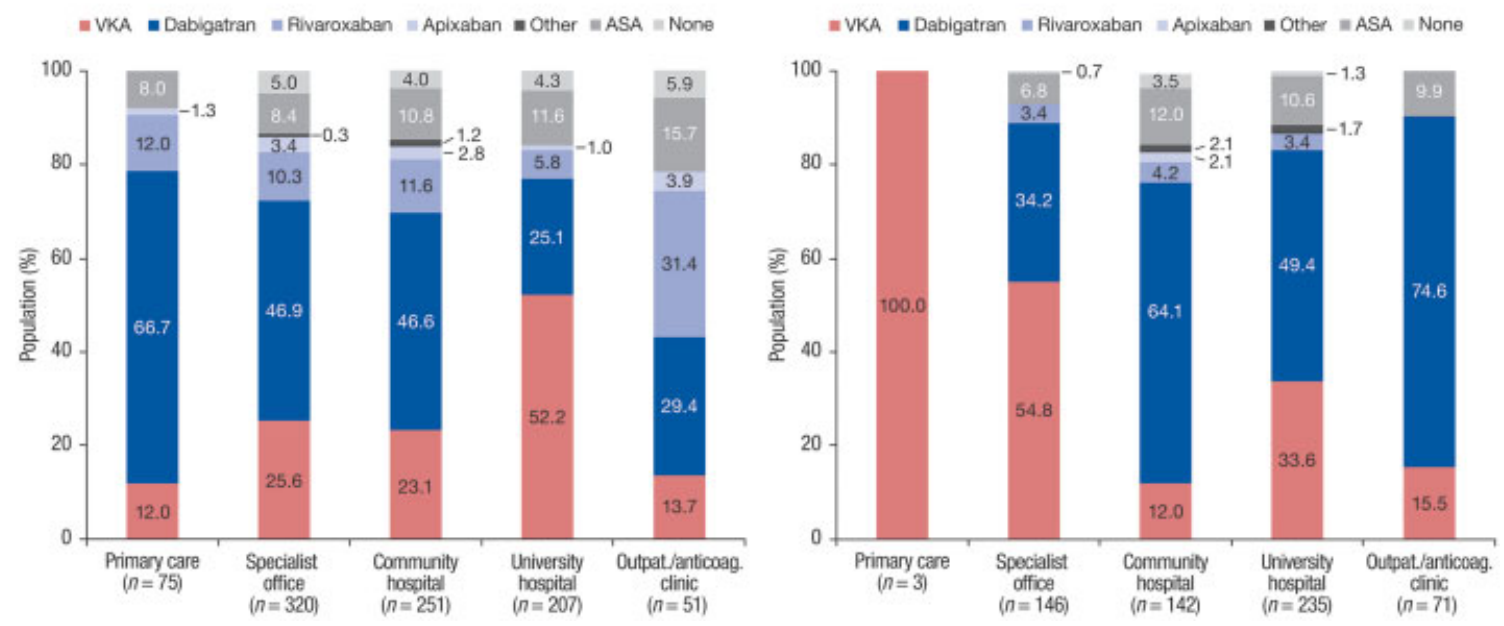

Fig. 4 Antithrombotic treatment by region and setting. ${ }^{\text {a }}$ Other = antiplatelets other than acetylsalicylic acid (ASA) and combination of antithrombotic

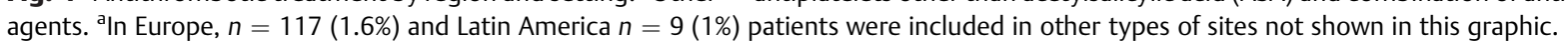

probability ratios were calculated for OAC use versus other therapy use.

A lower adjusted probability for OAC treatment prescription compared with other therapy was found for patients with low and moderate thromboembolic risk by $\mathrm{CHA}_{2} \mathrm{DS}_{2}$-VASc score (probability 0.63 [95\% CI: 0.55-0.71],
0.91 [95\% CI: 0.88-0.94], respectively) and high bleeding risk by HAS-BLED score (0.79 [95\% CI: 0.76-0.82]) as well as those recruited in regions of Asia, North and Latin America (vs. Europe, probability ratios 0.66 [95\% CI: 0.640.69], 0.91 [95\% CI: 0.89-0.93], 0.96 [95\% CI: 0.94-0.99], respectively). 


\section{Non-Vitamin K Antagonist versus Vitamin K Antagonist}

A log-binomial regression analysis for estimation of probability ratios for the prescription of NOAC versus VKA is presented in -Supplementary Table S2 (online only). In the multivariable analysis, adjusted probability ratios were calculated for NOAC (compared with VKA use).

Patients recruited at specialist office, community hospital and other health care settings, compared with university hospitals, had a higher adjusted probability for NOAC versus VKA prescription, probability ratios 1.53 (95\% CI: 1.46-1.60), 1.46 (95\% CI: 1.39-1.53), 1.52 (95\% CI: 1.43-1.60), respectively. In contrast, patients with persistent $\mathrm{AF}$ (vs. paroxysmal $\mathrm{AF}$, probability ratio 0.93 [95\% CI: 0.90-0.96]), diabetes mellitus (probability ratio 0.92 [95\% CI: 0.88-0.96]), prior myocardial infarction (probability ratio 0.85 [95\% CI: $0.80-0.91]$ ) and chronic kidney disease (probability ratio 0.92 [95\% CI: 0.89-0.96]) had a lower probability for NOAC use, compared with VKA.

\section{Discussion}

The principal findings of this analysis are as follows: (1) despite a relatively high global OAC use, substantial interregional differences are evident, with $\mathrm{OAC}$ use being highest in Europe (90.1\%) and lowest in Asia (55.2\%); (2) among OAC users, NOACs have replaced VKAs as the more prevalent option in all regions, with the highest uptakes in North America (66.5\%) and lowest in Asia (50.2\%); (3) intra-regional differences in antithrombotic therapy use are apparent; and (4) AF guideline adherence requires improvement, as nearly half of low-risk patients are over-treated, while every sixth high-risk patient (8.8\% in Europe, $18.9 \%$ in North America and $42.4 \%$ in Asia) is under-treated with OAC.

There may be several potential reasons for substantial heterogeneity in OAC use by regions in this analysis, such as apparent differences in baseline characteristics, associated comorbid disease, thromboembolic and bleeding risk, type of AF or enrolling site, physicians' specialty, AF guidelines or health care system and reimbursement in a given country. ${ }^{11-13,22-24}$ While some previous reports have shown that there are regions with lower OAC uptake, particularly in Asia, we found that the uptake within regions varies considerably by country, health care setting and type of anticoagulation (VKA vs. NOACs). ${ }^{25-27}$

Prior studies have reported several issues limiting optimal anticoagulation, such as fear of bleeding complications or poor quality of INR control. ${ }^{27-29}$ Our study shows that NOACs are a more prevalent treatment option than VKAs in all study regions, and in 33 out of 44 participating countries. Even more importantly, higher anticoagulation rates were observed in those regions and countries where NOACs were the main anticoagulants. This was evident in Africa/Middle East and Latin America, which as regions was found to be associated with higher use of NOACs (vs. VKAs) compared with Europe, and reported even higher anticoagulation uptake than North America. Interestingly, in Africa/Middle East and Latin America, there was no reimbursement for medications in 14 to $26 \%$, while self-pay for medications was only reported in approximately 3 to $4 \%$ in North America and Europe.
Despite having the highest NOAC uptake among OAC users, overall anticoagulation use in North America was relatively low. This pattern may be partially explained by American AF guidelines, which in contrast to the European and Asia Pacific guidelines offer $\mathrm{OAC}$, aspirin or even no AF stroke prophylaxis in patients with $\mathrm{CHA}_{2} \mathrm{DS}_{2}$-VASc score of 1 , while $\mathrm{OAC}$ is recommended in those with the score $\geq 2 .^{30-32}$ When compared with other regions of GLORIA-AF registry, the proportion of participating cardiologists in North America was lowest. Indeed, lower prescription rates have been reported by registries where broader spectrum of physician specialties were included. ${ }^{24}$ In addition, the incidence of paroxysmal AF (as compared with other AF patterns) was also highest in North America and in the present analysis, was associated with lower OAC prescription. Other studies have also reported less OAC use in patients with paroxysmal AF, although current guidelines recommend anticoagulation regardless of AF pattern. ${ }^{30,33}$

The highest overall OAC use was reported in Europe, with generally NOACs being more frequently prescribed than VKAs. However in some countries, including high-enrolling ones (i.e., the Netherlands, Spain and United Kingdom), VKAs were more frequently prescribed than NOACs. Western European countries generally provide good quantity and quality of anticoagulation and more guideline-adherent OAC use compared with other European and non-European countries. ${ }^{16,34,35}$ Regardless of OAC type (whether with an NOAC or VKA), being treated for AF in Europe was associated with higher likelihood of being anticoagulated (vs. antiplatelet or no therapy), compared with North and Latin America or Asia.

Another important finding relates to health care clinical settings. Overall physicians from university hospitals prescribed anticoagulation less frequently than those from other health care settings, and preferred VKAs over NOACs. Potential reasons for such differences in treatment patterns by setting may be multifactorial and may include more common and complex invasive procedures being performed or higher number of patients with comorbid disease and contraindications to OAC being enrolled by university hospitals. Nonetheless, a growing body of evidence suggests that OAC should be neither interrupted nor bridged during various invasive procedures, including AF ablation, as this does not lower the risk of bleeding complications but brings increased risk of thromboembolism. ${ }^{36-38}$

Geographic differences may also play a role. For example, OAC prescription in Asian university hospitals was nearly twofold lower than in community hospitals or specialist offices, whereas in other regions a more homogenous distribution of OAC by setting (regardless of anticoagulant type) was observed. Such heterogeneity in anticoagulation rates between community and university hospitals in Asia may reside in more evident between-country differences in national health care systems compared with other geographic regions. In addition, country- and site-specific OAC prescription patterns and predominantly enrolling sites may play a role. Indeed, for example, 1 in 5 patients in China versus 9 in 10 in Japan were prescribed OAC. A marked difference in NOAC versus VKA prescription was also noted, with overall higher OAC use in those Asian countries where NOAC was the main anticoagulant. 
In Europe, nearly two-thirds of low-risk patients were anticoagulated, while many patients with $\geq 2$ stroke risk factors are not treated as such. ${ }^{39,40}$ Also, aspirin is commonly prescribed to low-risk patients and those at high risk of bleeding, being prescribed in $10.0 \%$ of subjects at high thromboembolic risk. Stroke prevention with aspirin monotherapy is neither effective nor safe, and majority of AF guidelines do not recommend its use. ${ }^{30,32,41}$ Such non-compliance with guidelines has been reported by previous registries, which observed improved outcomes in patients who were treated in line with AF guidelines. ${ }^{14,16}$

\section{Limitations and Strengths}

GLORIA-AF is not a population-based programme, which limits patient representativeness. Particularly, relatively low number of patients has been enrolled from Latin America and Africa/Middle East. Patients could enter the study only after signing an informed consent, which might have resulted in higher anticoagulation rates compared with general AF population. Inclusion of patients with new onset $A F$ and once NOACs were on offer in given countries could result in higher OAC rates compared with registries which enroll AF 'allcomers'. As countries with various health care systems, drug reimbursement policy, predominantly enrolling sites, $\mathrm{AF}$ guidelines and different OAC prescribing patterns have been grouped within one geographic region, a bias resulting from a direct comparison of antithrombotic treatment among such defined regions cannot be excluded. Because a considerable number of countries did not enrol enough patients to allow meaningful country-specific data, country (as a variable) was not incorporated into the multivariable analysis. Higher use of dabigatran versus other NOACs should not be surprising as this report covers specifically the period that started once the first NOAC (predominately dabigatran) became available in a given country. ${ }^{17,42}$

Nevertheless, despite not being representative for the general AF population, GLORIA-AF is as much representative as other AF registries in the field, what is also reflected by inclusion/exclusion criteria. Global reach, broad country, site and physician inclusion, high-quality control measures along with unique study design with phase II commencing data collection immediately following first NOAC launch in given countries are evident strengths of GLORIA-AF. Consequently, this report provides an up-to-date global, regional (continental) and local (including country and health care setting) overview of contemporary antithrombotic treatment patterns for stroke prevention in newly diagnosed AF.

\section{Conclusion}

Substantial inter- and intra-regional differences in ATT for stroke prevention in AF are evident, as summarized in - Fig. $\mathbf{5}$. While guideline-adherent ATT can be further improved, NOACs are the main contributor to high OAC use worldwide.

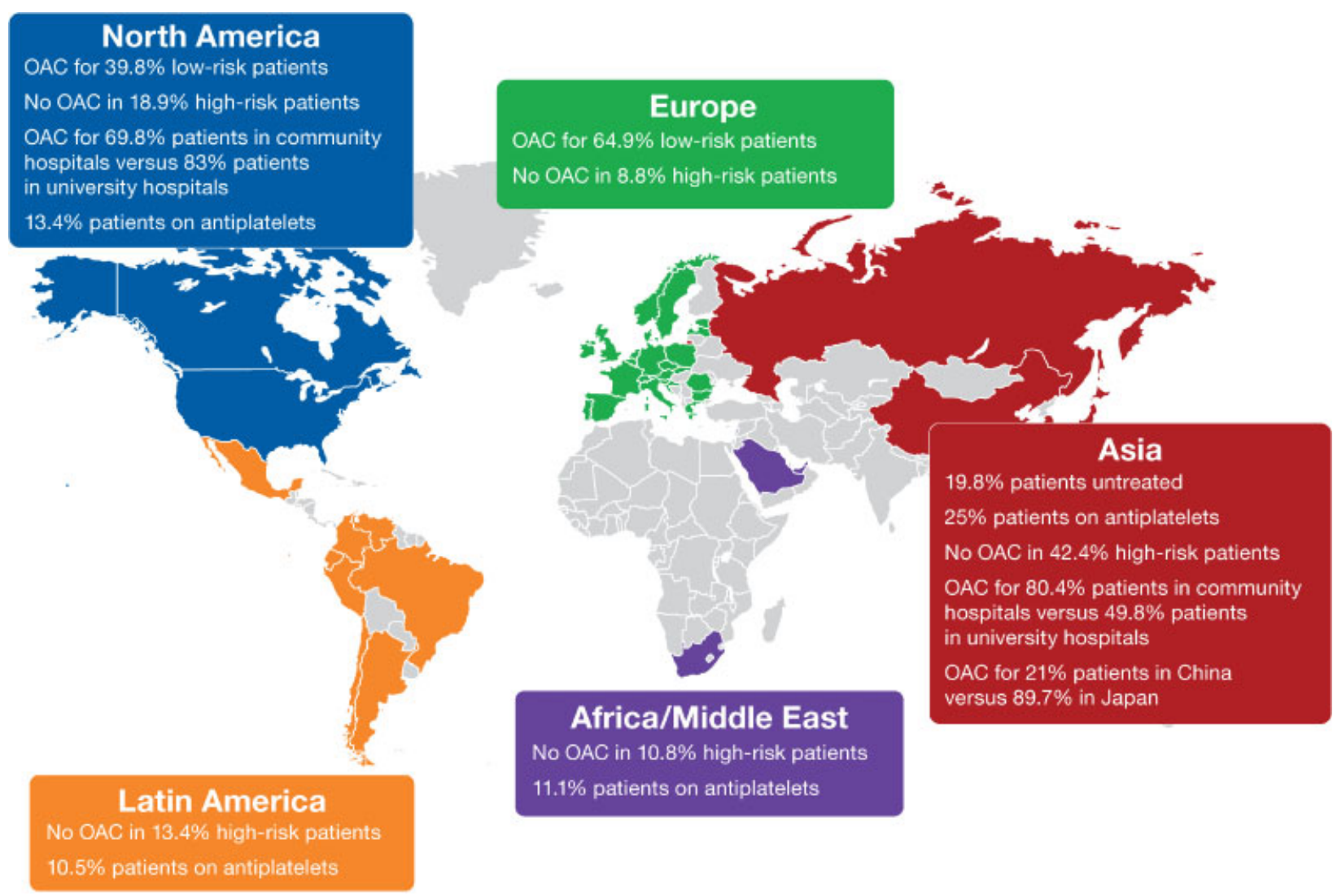

Fig. 5 Summary infographic showing substantial inter- and intra-regional differences in antithrombotic therapy for stroke prevention in AF (atrial fibrillation). 


\section{What is known about this topic?}

- Although guideline-adherent antithrombotic therapy (ATT) for stroke prevention in atrial fibrillation (AF) is associated with lower mortality and thromboembolism, ATT uptake for AF shows geographic variation worldwide.

\section{What does this paper add?}

- In this global registry of 15,092 patients newly diagnosed with non-valvular AF at risk for stroke, oral anticoagulation (OAC) use was $79.9 \%$, being highest in Europe (90.1\%), followed by Africa/Middle East (87.4\%), Latin America (85.3\%), North America (78.3\%) and Asia (55.2\%).

- Among OAC users, vitamin K antagonists (VKAs) have been replaced by non-VKA OACs (NOACs) as the more prevalent OAC option in all regions, with highest use in North America (66.5\%) and lowest use in Asia (50.2\%).

- Globally, 76.5\% of low-risk patients were prescribed ATT (46.1\% OAC), whereas $17.7 \%$ high-risk patients were not anticoagulated (Europe 8.8\%, North America 18.9\% and Asia 42.4\%).

- Substantial inter- and intra-regional differences in ATT for stroke prevention in AF are evident.

\section{Conflicts of Interest}

M.M. declared no conflict of interest. M.V.H. has received honoraria for presentations and research grants from Boehringer Ingelheim, Bayer HealthCare, Pfizer, GlaxoSmithKline (GSK) and Actelion Pharmaceuticals. K.J.R. is an employee of RTI Health Solutions, an independent non-profit research organization that does work for government agencies and pharmaceutical companies. S.J.D. has received consultancy fees for serving as a steering committee member for Boehringer Ingelheim. He also holds research grants from St Jude Medical. C.S.M. has received honoraria for presentations as well as research grants from BMS, Boehringer Ingelheim, Bayer HealthCare, Pfizer, AstraZeneca and Johnson \& Johnson. H.C.D. has received honoraria for participation in clinical trials, contribution to advisory boards or oral presentations from the following: Abbott, Allergan, AstraZeneca, Bayer Vital, BMS, Boehringer Ingelheim, CoAxia, Corimmun, Covidien, Daiichi-Sankyo, D-Pharm, Fresenius, GSK, Janssen-Cilag, Johnson \& Johnson, Knoll, Lilly, MSD, Medtronic, MindFrame, Neurobiological Technologies, Novartis, Novo-Nordisk, Paion, Parke-Davis, Pfizer, SanofiAventis, Schering-Plough, Servier, Solvay, St Jude, Syngis, Talecris, Thrombogenics, WebMD Global, Wyeth and Yamanouchi. Financial support for research projects was provided by AstraZeneca, GSK, Boehringer Ingelheim, Lundbeck, Novartis, Janssen-Cilag, Sanofi-Aventis, Syngis and Talecris. The Department of Neurology at the University Duisburg-Essen received research grants from the German Research Council (DFG), the German Ministry of Education and Research
(BMBF), the European Union, the National Institutes of Health (NIH), the Bertelsmann Foundation and the HeinzNixdorf Foundation. H.C.D. has no ownership interest and does not own stocks of any pharmaceutical company; within the past year, he served as the editor of Aktuelle Neurologie, Arzneimitteltherapie, Kopfschmerznews, Stroke News, as the co-editor of Cephalalgia and was on the editorial board of Lancet Neurology, Stroke, European Neurology and Cerebrovascular Disorders; he chairs the Treatment Guidelines Committee of the German Society of Neurology and has contributed to the European Heart Rhythm Association (EHRA) and the European Society of Cardiology (ESC) guidelines for the treatment of atrial fibrillation. J.L.H. is currently conducting research sponsored by Boehringer Ingelheim as a member of the Executive Steering Committee for the GLORIA-AF Registry, and has received consulting fees from Bayer HealthCare, Janssen-Ortho-McNeil, and Pfizer for advisory activities involving the development of anticoagulant drugs. M.P., S.L., K.Z., C.T. and A.E. are employees of Boehringer Ingelheim. G.Y.H.L. has been a consultant for Bayer/Janssen, Astellas, Merck, Sanofi, Bristol-Myers Squibb (BMS)/Pfizer, Biotronik, Medtronic, Portola, Boehringer Ingelheim, Microlife and Daiichi-Sankyo. He has also been a speaker for Bayer, BMS/Pfizer, Medtronic, Boehringer Ingelheim, Microlife, Roche and Daiichi-Sankyo.

\section{Authorship}

All authors had access to the data and played a role in writing this article.

Funding

This work was supported by Boehringer Ingelheim $\mathrm{GmbH}$.

Clinical Trial Registration

http://www.clinicaltrials.gov.

Unique identifier: NCT01468701.

\section{References}

1 Benjamin EJ, Wolf PA, D'Agostino RB, Silbershatz H, Kannel WB, Levy D. Impact of atrial fibrillation on the risk of death: the Framingham Heart Study. Circulation 1998;98(10):946952

2 Stewart S, Hart CL, Hole DJ, McMurray JJV. A population-based study of the long-term risks associated with atrial fibrillation: 20-year follow-up of the Renfrew/Paisley study. Am J Med 2002; 113(05):359-364

3 Miyasaka Y, Barnes ME, Bailey KR, et al. Mortality trends in patients diagnosed with first atrial fibrillation: a 21-year community-based study. J Am Coll Cardiol 2007;49(09):986-992

4 Hart RG, Pearce LA, Aguilar MI. Meta-analysis: antithrombotic therapy to prevent stroke in patients who have nonvalvular atrial fibrillation. Ann Intern Med 2007;146(12):857-867

5 Connolly SJ, Ezekowitz MD, Yusuf S, et al; RE-LY Steering Committee and Investigators. Dabigatran versus warfarin in patients with atrial fibrillation. N Engl J Med 2009;361(12): 1139-1151

6 Patel MR, Mahaffey KW, Garg J, et al; ROCKET AF Investigators. Rivaroxaban versus warfarin in nonvalvular atrial fibrillation. N Engl J Med 2011;365(10):883-891 
7 Granger CB, Alexander JH, McMurray JJV, et al; ARISTOTLE Committees and Investigators. Apixaban versus warfarin in patients with atrial fibrillation. N Engl J Med 2011;365(11):981-992

8 Giugliano RP, Ruff CT, Braunwald E, et al; ENGAGE AF-TIMI 48 Investigators. Edoxaban versus warfarin in patients with atrial fibrillation. N Engl J Med 2013;369(22):2093-2104

9 Ruff CT, Giugliano RP, Braunwald E, et al. Comparison of the efficacy and safety of new oral anticoagulants with warfarin in patients with atrial fibrillation: a meta-analysis of randomised trials. Lancet 2014;383(9921):955-962

10 Freedman B, Potpara TS, Lip GY. Stroke prevention in atrial fibrillation. Lancet 2016;388(10046):806-817

11 Gamra H, Murin J, Chiang CE, Naditch-Brûlé L, Brette S, Steg PG; RealiseAF Investigators. Use of antithrombotics in atrial fibrillation in Africa, Europe, Asia and South America: insights from the International RealiseAF Survey. Arch Cardiovasc Dis 2014;107 (02):77-87

12 Le Heuzey JY, Ammentorp B, Darius H, et al. Differences among western European countries in anticoagulation management of atrial fibrillation. Data from the PREFER IN AF registry. Thromb Haemost 2014;111(05):833-841

13 Lip GY, Laroche C, Boriani G, et al. Regional differences in presentation and treatment of patients with atrial fibrillation in Europe: a report from the EURObservational Research Programme Atrial Fibrillation (EORP-AF) Pilot General Registry. Europace 2015;17(02):194-206

14 Nieuwlaat R, Olsson SB, Lip GY, et al; Euro Heart Survey Investigators; The Euro Heart Survey on Atrial Fibrillation. Guideline-adherent antithrombotic treatment is associated with improved outcomes compared with undertreatment in high-risk patients with atrial fibrillation. Am Heart J 2007;153 (06):1006-1012

15 Chao T-FF, Liu C-JJ, Tuan T-CC, et al. Impact on Outcomes of Changing Treatment Guideline Recommendations for Stroke Prevention in Atrial Fibrillation: a nationwide cohort study. Mayo Clin Proc 2016;91(05):567-574

16 Lip GY, Laroche C, Popescu MI, et al. Improved outcomes with European Society of Cardiology guideline-adherent antithrombotic treatment in high-risk patients with atrial fibrillation: a report from the EORP-AF General Pilot Registry. Europace 2015; 17(12):1777-1786

17 Huisman MV, Lip GY, Diener HC, et al. Design and rationale of Global Registry on Long-Term Oral Antithrombotic Treatment in Patients with Atrial Fibrillation: a global registry program on long-term oral antithrombotic treatment in patients with atrial fibrillation. Am Heart J 2014;167(03):329-334

18 Lip GY, Nieuwlaat R, Pisters R, Lane DA, Crijns HJ. Refining clinical risk stratification for predicting stroke and thromboembolism in atrial fibrillation using a novel risk factor-based approach: the euro heart survey on atrial fibrillation. Chest 2010;137(02): 263-272

19 Pisters R, Lane DA, Nieuwlaat R, de Vos CB, Crijns HJ, Lip GY. A novel user-friendly score (HAS-BLED) to assess 1-year risk of major bleeding in patients with atrial fibrillation: the Euro Heart Survey. Chest 2010;138(05):1093-1100

20 McNutt L-A, Wu C, Xue X, Hafner JP. Estimating the relative risk in cohort studies and clinical trials of common outcomes. Am J Epidemiol 2003;157(10):940-943

21 Deddens JA, Petersen MR. Approaches for estimating prevalence ratios. Occup Environ Med 2008;65(07):481, 501-506

22 Camm AJ, Pinto FJ, Hankey GJ, Andreotti F, Hobbs FD; Writing Committee of the Action for Stroke Prevention alliance. Nonvitamin $\mathrm{K}$ antagonist oral anticoagulants and atrial fibrillation guidelines in practice: barriers to and strategies for optimal implementation. Europace 2015;17(07):1007-1017

23 Fosbol EL, Holmes DN, Piccini JP, et al; ORBIT-AF Investigators and Patients. Provider specialty and atrial fibrillation treatment stra- tegies in United States community practice: findings from the ORBIT-AF registry. J Am Heart Assoc 2013;2(04):e000110

24 Kakkar AK, Mueller I, Bassand J-P, et al; GARFIELD Registry Investigators. Risk profiles and antithrombotic treatment of patients newly diagnosed with atrial fibrillation at risk of stroke: perspectives from the international, observational, prospective GARFIELD registry. PLoS One 2013;8(05):e63479

25 Huisman MV, Ma CS, Diener H-C, et al; GLORIA-AF Investigators. Antithrombotic therapy use in patients with atrial fibrillation before the era of non-vitamin $\mathrm{K}$ antagonist oral anticoagulants: the Global Registry on Long-Term Oral Antithrombotic Treatment in Patients with Atrial Fibrillation (GLORIA-AF) Phase I cohort. Europace 2016;18(09):1308-1318

26 Siu C-W, Lip GY, Lam K-F, Tse H-F. Risk of stroke and intracranial hemorrhage in 9727 Chinese with atrial fibrillation in Hong Kong. Heart Rhythm 2014;11(08):1401-1408

27 Akao M, Chun Y-H, Esato M, et al; Fushimi AF Registry Investigators. Inappropriate use of oral anticoagulants for patients with atrial fibrillation. Circ J 2014;78(09):2166-2172

28 Atarashi H, Inoue H, Okumura K, Yamashita T, Kumagai N, Origasa $\mathrm{H}$; J-RHYTHM Registry Investigators. Present status of anticoagulation treatment in Japanese patients with atrial fibrillation: a report from the J-RHYTHM Registry. Circ J 2011;75(06): 1328-1333

29 Chiang C-E, Wang K-L, Lip GY. Stroke prevention in atrial fibrillation: an Asian perspective. Thromb Haemost 2014;111 (05):789-797

30 Kirchhof P, Benussi S, Kotecha D, et al. 2016 ESC Guidelines for the management of atrial fibrillation developed in collaboration with EACTS. Eur Heart J 2016;37(38):2893-2962

31 January CT, Wann LS, Alpert JS, et al; ACC/AHA Task Force Members. 2014 AHA/ACC/HRS guideline for the management of patients with atrial fibrillation: a report of the American College of Cardiology/American Heart Association Task Force on practice guidelines and the Heart Rhythm Society. Circulation 2014;130 (23):e199-e267

32 Ogawa S, Aonuma K, Tse HF, et al. The APHRS's 2013 statement on antithrombotic therapy of patients with nonvalvular atrial fibrillation. Journal of Arrhythmia 2013;29(03):190-200

33 Nieuwlaat R, Dinh T, Olsson SB, et al; Euro Heart Survey Investigators. Should we abandon the common practice of withholding oral anticoagulation in paroxysmal atrial fibrillation? Eur Heart J 2008;29(07):915-922

34 Kirchhof P, Ammentorp B, Darius H, et al. Management of atrial fibrillation in seven European countries after the publication of the 2010 ESC Guidelines on atrial fibrillation: primary results of the PREvention oF thromboemolic events-European Registry in Atrial Fibrillation (PREFER in AF). Europace 2014;16(01): 6-14

35 Singer DE, Hellkamp AS, Piccini JP, et al; ROCKET AF Investigators. Impact of global geographic region on time in therapeutic range on warfarin anticoagulant therapy: data from the ROCKET AF clinical trial. J Am Heart Assoc 2013;2(01):e000067

36 Di Biase L, Burkhardt JD, Santangeli P, et al. Periprocedural stroke and bleeding complications in patients undergoing catheter ablation of atrial fibrillation with different anticoagulation management: results from the Role of Coumadin in Preventing Thromboembolism in Atrial Fibrillation (AF) Patients Undergoing Catheter Ablation (COMPARE) randomized trial. Circulation 2014; 129(25):2638-2644

37 Zhao Y, Yang Y, Tang X, Yu X, Zhang L, Xiao H. New oral anticoagulants compared to warfarin for perioperative anticoagulation in patients undergoing atrial fibrillation catheter ablation: a meta-analysis of continuous or interrupted new oral anticoagulants during ablation compared to interrupted or continuous warfarin. J Interv Card Electrophysiol 2017;48 (03):267-282 
38 Calkins H, Willems S, Gerstenfeld EP, et al; RE-CIRCUIT Investigators. Uninterrupted dabigatran versus warfarin for ablation in atrial fibrillation. N Engl J Med 2017;376(17):1627-1636

39 Lip GY, Nielsen PB. Should patients with atrial fibrillation and 1 stroke risk factor (CHA2DS2-VASc score 1 in men, 2 in women) be anticoagulated? Yes: even 1 stroke risk factor confers a real risk of stroke. Circulation 2016;133(15):1498-1503, discussion 1503

40 Lip GY, Skjøth F, Rasmussen LH, Nielsen PB, Larsen TB. Net clinical benefit for oral anticoagulation, aspirin, or no therapy in nonvalvular atrial fibrillation patients with 1 additional risk factor of the CHA2DS2VASc score (beyond sex). J Am Coll Cardiol 2015;66(04):488-490

41 National Institute for Health and Care Excellence. Atrial Fibrillation: The Management of Atrial Fibrillation. Available at: http:// www.ncbi.nlm.nih.gov/pubmed/25340239. Accessed September 12,2016

42 Huisman MV, Rothman KJ, Paquette M, et al; GLORIA-AF Investigators. Antithrombotic treatment patterns in patients with newly diagnosed nonvalvular atrial fibrillation: the GLORIA-AF registry, phase II. Am J Med 2015;128(12):1306-1313.e1 